\title{
Calcium-dependent mitochondrial permeability transition is augmented in the kidney of Goto-Kakizaki diabetic rat
}

\author{
Paulo J. Oliveira ${ }^{1 *}$ \\ Telma C. Esteves ${ }^{1,2}$ \\ Raquel Seiça ${ }^{3}$ \\ António J. M. Moreno ${ }^{1}$ \\ Maria S. Santos ${ }^{1}$ \\ ${ }^{1}$ Centro de Neurociências de Coimbra, \\ Departamento de Zoologia, Faculdade \\ de Ciências e Tecnologia, \\ Universidade de Coimbra, Portugal \\ ${ }^{2}$ Departamento de Biologia Vegetal, \\ Faculdade de Ciências, Universidade \\ de Lisboa, Portugal
}

${ }^{3}$ Faculdade de Medicina, Centro de Neurociências de Coimbra, Universidade de Coimbra, Portugal

*Correspondence to: Paulo J. Oliveira, Centro de Neurociências de Coimbra, Departamento de Zoologia, Universidade de Coimbra, P-3004-517 Coimbra, Portugal. E-mail: pauloliv@ci.uc.pt
Received: 24 April 2003

Revised: 1 August 2003

Accepted: 6 October 2003

\begin{abstract}
Background Renal disease associated with diabetes mellitus is a major problem among diabetic patients. The role of mitochondria in the pathogenesis of diabetes has received a large amount of attention in the last years, but many aspects of this subject are still poorly understood. In the present study, we studied the susceptibility of the mitochondrial permeability transition (MPT) on kidney mitochondria from the Goto-Kakizaki (GK) rat, an animal model featuring physiological and pathological alterations characteristic of type 2 diabetes.
\end{abstract}

Methods Kidney mitochondria were isolated by differential centrifugations; mitochondrial electric transmembrane potential and calcium loading capacity were evaluated with a $\mathrm{TPP}^{+}$-selective electrode and with a calcium-sensitive fluorescent probe. Coenzyme Q9, Q10 and vitamin E were evaluated by high-performance liquid chromatography (HPLC).

Results Kidney mitochondria from the diabetic animals had an increased susceptibility to the induction of the MPT by calcium. We observed a loss of calcium-loading capacity and a higher calcium-induced mitochondrial depolarization. Vitamin E and coenzyme Q9 were also increased in kidney mitochondria from GK rats.

Conclusions The results show an enhanced MPT activation in kidney mitochondria from GK rats, which lead us to suggest that this condition may be one major alteration triggered by chronic diabetes in kidney cells, ultimately leading to cell dysfunction. Copyright $\odot 2004$ John Wiley \& Sons, Ltd.

Keywords mitochondrial permeability transition; kidney mitochondria; type 2 diabetes; Goto-Kakizaki rat

\section{Introduction}

Type 2 diabetes is the most common type of diabetes nowadays, accounting for nearly $90 \%$ of all known cases. Among other complications, diabetes mellitus-associated renal disease is a major concern among diabetic patients $[1,2]$.

Knowledge about the nature of the mitochondrial permeability transition (MPT) and its precise physiological and pathological role in a chronic disease such as type 2 diabetes is scarce. Relating to kidney mitochondria, the information regarding MPT induction is totally unavailable. The MPT is 
triggered by the formation of a calcium and redoxsensitive pore complex from several mitochondrial proteins (MPTP). The MPT is known to be specifically inhibited by cyclosporin A [3]. MPT induction can lead to mitochondrial swelling, loss of mitochondrial membrane potential and loss of calcium-loading capacity [4,5].

Animal models for type 2 diabetes, such as the Goto-Kakizaki (GK) rat, are important to the study of biochemical changes associated with diabetes [6]. The GK rat is an inbreed rat strain developed by selective breeding with glucose intolerance as a selection index of an outbreed colony of non-diabetic Wistar strain rats, being a well-characterised model for non-obese type 2 diabetes [6]. This particular diabetic model develops characteristic tissue damage in several organs (including the kidney) that resemble systemic manifestations encountered in human type 2 diabetes [6]. This animal model may be particularly useful in studying mitochondrial alterations during the course of the disease.

The present study analysed the mitochondrial susceptibility to calcium-induced MPT in kidney mitochondria from GK and control Wistar rats with the same age. We were expecting to find differences regarding the calciumloading capacity in kidney mitochondria of GK rats. We also measured the mitochondrial amounts of coenzyme Q9, Q10 and vitamin E in order to obtain further insights of adaptations suffered by kidney mitochondria to the disease condition, as those molecules are known to be involved in mitochondrial antioxidant defences.

\section{Experimental procedures}

\section{Animal care}

Twenty-six-week-old male GK rats were obtained from our breeding colony raised from couples kindly offered to our laboratory by Dr K. I. Susuki (Tohoku University, School of Medicine, Sendai, Japan). Male Wistar rats (26week old) were used as controls. All animals were raised under controlled temperature, humidity and light-dark cycle conditions, provided with access ad libitum to complete diet and drinking water. Animals were killed by cervical dislocation. The non-fasting blood glucose values were of $119.25 \pm 21.9 \mathrm{mg} / \mathrm{dL}$ for control animals and $294 \pm 83.4 \mathrm{mg} / \mathrm{dL}$ for diabetic GK rats $(p<0.005, n=$ $5)$. In this study, the 'Principles of Laboratory animal care' (NIH publication $n^{\circ} 83-25$, revised 1985) were followed.

\section{Chemicals}

All chemicals and reagents used were of the highest grade of purity commercially available. Calcium Green 5-N was from Molecular Probes, Eugene, OR.

\section{Isolation of kidney mitochondria}

After removing the capsule and the renal medulla, the kidneys' cortex was minced finely in an ice-cold isolation medium containing 250-mM sucrose, 10-mM HEPES$\mathrm{KOH}, 1-\mathrm{mM}$ EGTA and $0.1 \%$ BSA lipid free, adjusted to $\mathrm{pH} 7.4$ with $\mathrm{KOH}$. Minced blood-free tissue was homogenised with a tightly fitted homogeniser (Teflonglass pestle) and then centrifuged at $800 \mathrm{~g}$ for $10 \mathrm{~min}$ at $4{ }^{\circ} \mathrm{C}$. The supernatant fluid was retained and centrifuged at $10000 \mathrm{~g}$ for $10 \mathrm{~min}$. The pellet was resuspended using a paint brush and repelleted twice at 10000 for $10 \mathrm{~min}$ in washing medium containing 250-mM sucrose and 10-mM HEPES. Sorvall RC-5C Plus (SS 34 rotor) was used for centrifugations. Following the final wash, mitochondria were resuspended in $1 \mathrm{~mL}$ of the washing medium. Mitochondrial protein was assayed by the biuret method with BSA as standard. No significant changes in mitochondrial isolation yield were observed.

\section{Measurement of mitochondrial membrane potential}

Mitochondrial membrane potential $(\Delta \psi)$ was measured with a tetraphenylphosphonium cation $\left(\mathrm{TPP}^{+}\right)$electrode, as described by Kamo et al. [7]. No correction for the passive binding contribution of $\mathrm{TPP}^{+}$was used, as the purpose of this study was to show the relative changes in the potential, rather than absolute values. A mitochondrial matricial volume of $1.1 \mu \mathrm{L} / \mathrm{mg}$ of protein was assumed. Reactions were carried out in $2 \mathrm{~mL}$ of medium containing 200-mM sucrose, 10-mM TRIS, 10- $\mu$ M EGTA and 1-mM $\mathrm{KH}_{2} \mathrm{PO}_{4}$ (pH 7.2), supplemented with $1-\mu \mathrm{M} \mathrm{TPP}{ }^{+}$, 4$\mu \mathrm{M}$ rotenone and $0.5-\mu \mathrm{g}$ oligomycin $/ \mathrm{mL}$. Mitochondria (1 mg/mL) were energised with $8-\mathrm{mM}$ succinate.

\section{Mitochondrial $\mathrm{Ca}^{2+}$-induced calcium release}

The hexapotassium salt of the fluorescence probe Calcium Green 5-N was used to measure extramitochondrial free $\mathrm{Ca}^{2+}$ [8]. $0.6 \mathrm{mg}$ of protein was resuspended in $2 \mathrm{~mL}$ of medium containing 200-mM sucrose, 10-mM TRIS, 10- $\mu \mathrm{M}$ EGTA, 1-mM KH $\mathrm{KO}_{4}$, $4-\mu \mathrm{M}$ rotenone and $0.5-\mu \mathrm{g}$ oligomycin ( $\mathrm{pH} 7.4$ ). Free $\mathrm{Ca}^{2+}$ was monitored with $100-\mathrm{nM}$ Calcium Green 5-N. Fluorescence was recorded continuously in a waterjacketed cuvette holder at $25^{\circ} \mathrm{C}$ using a Perkin-Elmer LS50B fluorescence spectrophotometer with excitation and emission wavelengths of 506 and $531 \mathrm{~nm}$ respectively. Mitochondria were energised with $8-\mathrm{mM}$ succinate. Calibration of the calcium signal was performed adding known quantities of calcium to the media with uncoupled mitochondria.

\section{Extraction and quantification of CoQ9, CoQ10 and vitamin $E$}

Aliquots of mitochondria containing $1 \mathrm{mg}$ of protein/mL were extracted according to the method described by Takada et al. [9]. The extract was evaporated to dryness 
under a stream of $\mathrm{N}_{2}$ and resuspended in absolute ethanol. CoQ content was determined by reverse-phase HPLC (Spherisorb RP18, S5ODS2 column). Samples were eluted with methanol: heptane $(10: 2 \mathrm{vol} / \mathrm{vol})$ at a flow rate of $2 \mathrm{~mL} / \mathrm{min}$. Detection was performed by an ultraviolet (UV) detector at $269 \mathrm{~nm}$. Vitamin E was extracted and quantified by following the method described by Takayanagi et al. [10]. The extract was evaporated to dryness under a stream of $\mathrm{N}_{2}$ and resuspended in $n$ hexane. Vitamin E content was determined by reversephase HPLC $(4.6 \times 200 \mathrm{~mm}$; Spherisorb S10w column $)$. Samples were eluted with $n$-hexane modified with $0.9 \%$ methanol at a flow rate of $1.5 \mathrm{~mL} / \mathrm{min}$. Detection was performed by a UV detector at $287 \mathrm{~nm}$.

\section{Result presentation}

On the same day, one control and one diabetic animal were tested. Some of the results are presented as typical comparative recordings of each one of those days. Otherwise, they are presented as mean \pm SEM of the number of different preparations described. Comparisons between groups were made using One Way Analysis of Variance (ANOVA), two-tailed unpaired $t$-tests or ANOVA followed by the Newman-Keuls post-test. A $p$ value $<0.05$ was considered statistically significant.

\section{Results}

The measurement of mitochondrial $\Delta \psi$ variations allowed us to indirectly assess the mitochondrial calcium susceptibility on both Wistar and GK kidney mitochondria. As seen in Figure 1(a) and (b), kidney mitochondria from Wistar rats presented higher values of membrane potential when measured $90 \mathrm{~s}$ after the addition of calcium $(-182.2 \pm 5.9 \mathrm{mV}$ in Wistar rats vs $-159.2 \pm 5.5 \mathrm{mV}$ in GK rats, $p<0.05)$.

The situation observed for GK mitochondria was compatible with MPTP opening, as it was inhibited by cyclosporin A, the specific pore inhibitor (trace GK + Cycl. A). In the presence of cyclosporin A, kidney mitochondria from GK rats restored the membrane potential to a value close to $-240 \mathrm{mV}$ (that is, equivalent to the initial values). This shows that the MPT is the cause for the reduction in mitochondrial calcium-loading capacity in the kidneys of GK rats. Cyclosporin A also inhibited the $\Delta \Psi$ decay observed after calcium addition to kidney mitochondria isolated from Wistar rats (membrane potential was restored to a value identical to the one in the GK group, $242.2 \pm 6.8 \mathrm{mV}$ for the GK group vs $249.5 \pm 3.4 \mathrm{mV}$ for the Wistar group, both in the presence of cyclosporin A).

Determination of extra-mitochondrial calcium movements using the fluorescent calcium-sensitive probe Calcium Green 5-N confirmed the data supplied by the TPP ${ }^{+}$. electrode experiments. The results showed that kidney mitochondria from Wistar rats had a higher calciumloading capacity than kidney mitochondria from GK rats
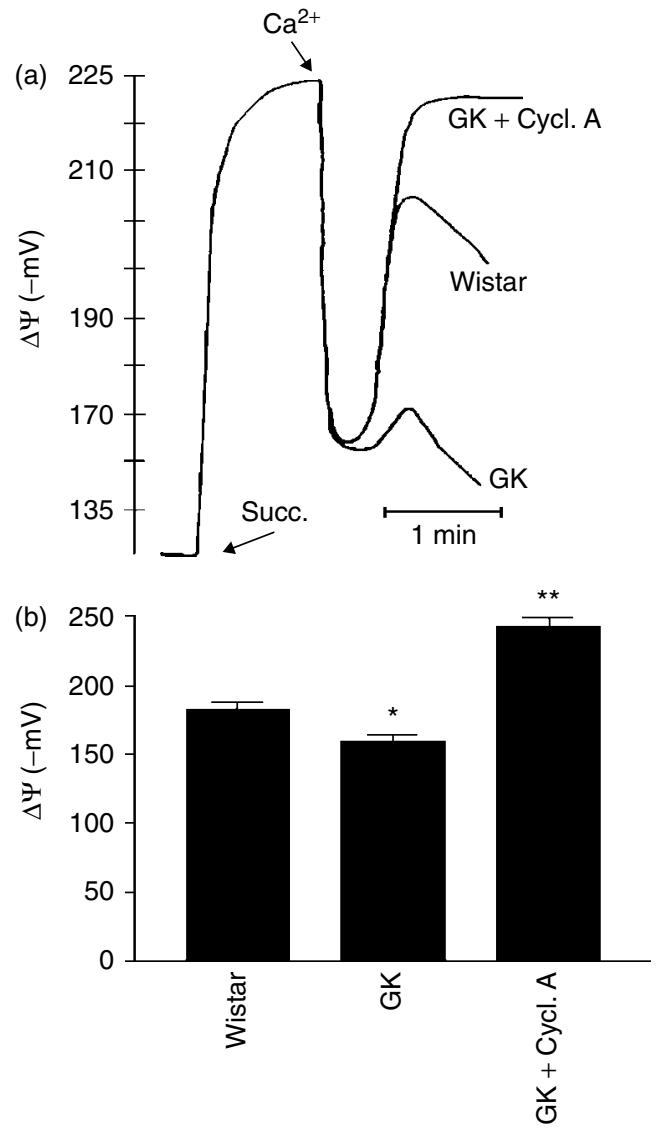

Figure 1. (a) Typical recording of mitochondrial electric potential measured by a TPP ${ }^{+}$-selective electrode. $250-\mu \mathrm{M} \mathrm{CaCl}_{2}$ was added in order to induce the MPTP. The $\Delta \Psi$ was calculated without $\mathrm{TPP}^{+}$-passive binding correction to mitochondrial membranes. Mitochondrial oxygen consumption and mitochondrial electric potential measurements were made simultaneously. A control assay was made with GK mitochondria and cyclosporin A (Cycl. A). For the sake of clarity, the recording correspondent to the control group in the presence of cyclosporin A is not shown. The recording shown here was typical of three different experiment days, with one animal of each group tested simultaneously. (b) Absolute values for mitochondrial membrane potential measured $90 \mathrm{~s}$ after calcium accumulation. Values are means \pm SEM of three different experiments. ${ }^{*} p<0.05$ vs Wistar group, ${ }^{* *} p<0.001$ vs GK group

before the overall loss of membrane impermeability characteristic of the MPT induction (Figure 2(a) and (b)). Figure 2(b) shows the amount of extra-mitochondrial calcium determined $300 \mathrm{~s}$ after calcium addition. Confirming the results presented in Figure 1, kidney mitochondria from Wistar rats were able to accumulate a higher amount of calcium than kidney mitochondria from GK rats. This fact was translated into a higher amount of extramitochondrial calcium in the GK group in the measured time end point $(4.23 \pm 1.36 \mathrm{nmol}$ vs $15.13 \pm 1.95 \mathrm{nmol}$, $p<0.001)$.

Cyclosporin A had a similar effect on both mitochondrial groups, increasing mitochondrial ability to accumulate calcium (values of extra-mitochondrial calcium after $300 \mathrm{~s}$ were $0.40 \pm 0.11 \mathrm{nmol}$ in the $\mathrm{GK}+$ cyclosporin $\mathrm{A}$ group vs $0.33 \pm 0.09 \mathrm{nmol}$ in the Wistar + cyclosporin A group). 

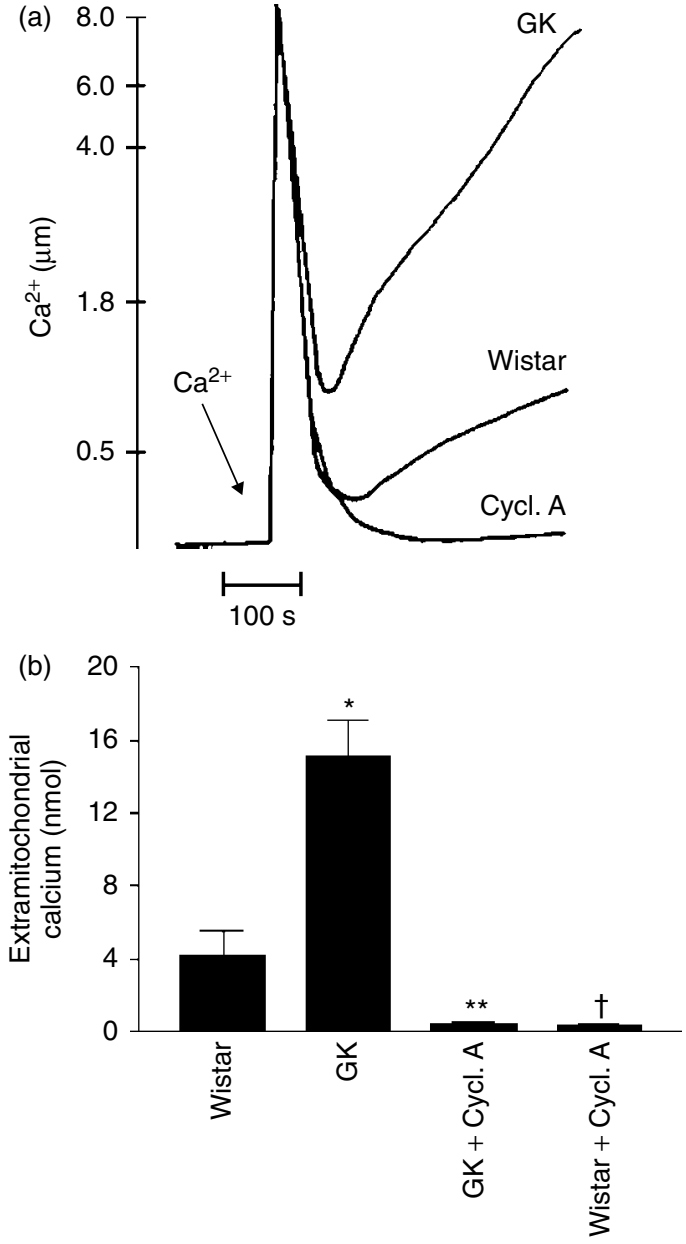

Figure 2. (a) Extra-mitochondrial calcium fluxes using the fluorescent calcium-sensitive probe Calcium Green 5-N. Ordinate quantifies the extra-mitochondrial calcium. EGTA was added at the end of each experiment to calculate the basal line. Extra-mitochondrial calcium was determined as described in the materials and methods section. A control assay was made with GK mitochondria and cyclosporin A. The recording shown here was typical of five different experiment days, with one animal of each group tested simultaneously. (b) Quantification of extra-mitochondrial calcium present in the buffer $300 \mathrm{~s}$ after calcium addition. Values are means \pm SEM of five different experiments. ${ }^{*} p<0.05$ vs Wistar group, ${ }^{* *} p<0.001$ vs GK group, $\dagger p<0.05$ vs Wistar group

We measured the mitochondrial amounts of coenzyme Q9, Q10 and vitamin E (Table 1). We found differences in the amounts of Vitamin E and coenzyme Q9, all increased in the GK group. Coenzyme Q10 was found without differences between both groups. In accordance with the differences observed, the ratio between coenzyme Q9 and Q10 as well as the sum of both coenzymes was also increased in the GK group.

\section{Discussion}

Although the MPTP is associated with important pathological situations, as well as with apoptotic and necrotic cell death [4], no studies were available on
Table 1. Determination of mitochondrial amounts of coenzyme Q9, conezyme Q10 and vitamin E, obtained as described before. Values are mean \pm SEM of 6 different preparations

\begin{tabular}{lrc}
\hline & Wistar group & \multicolumn{1}{c}{ GK group } \\
\hline Coenzyme Q9 (nmol/mg protein) & $4.84 \pm 0.28$ & $5.55 \pm 0.11^{*}$ \\
Coenzyme Q10 (nmol/mg protein) & $0.41 \pm 0.03$ & $0.35 \pm 0.02$ \\
Coenzyme Q10 + Q9 (nmol/mg protein) & $5.24 \pm 0.30$ & $5.9 \pm 0.12^{*}$ \\
Ratio Q9/Q10 & $11.97 \pm 0.50$ & $16.04 \pm 0.66^{*}$ \\
Vitamin E (nmol/mg protein) & $0.26 \pm 0.03$ & $0.43 \pm 0.04^{*}$ \\
\hline
\end{tabular}

${ }^{*} p<0.05$ vs Wistar group.

the induction of the MPTP in kidney mitochondria from diabetic GK rats.

The results presented in Figures 1 and 2 show that kidney mitochondria from GK rats have a lower calcium susceptibility. The equivalent effect of cyclosporin A on mitochondrial calcium susceptibility in both groups shows that the MPT was the sole reason for the reduced calcium uptake capacity in kidney mitochondria from the diabetic rats.

From the results, it is observable that mitochondria from control animals also suffered a certain degree of MPT induction. Probably, this happened in a more susceptible kidney mitochondrial population that depolarised and released calcium after MPT induction. Despite this, we consistently observed that the calcium-susceptible population seems to be increased in GK rats, although the membrane electric developed upon succinate addition (pre-calcium addition) was the same in both groups (see Figure 1(a)). The results suggest that kidney mitochondria from GK rats may act normally when maintaining homeostasis under resting conditions (precalcium) but may fail when subject to stress (for example, when accumulating calcium).

Although our experimental conditions may seem very artificial (specially concerning the high calcium concentrations used), it is known that microdomains may exist between mitochondria and the sarcoplasmic reticulum, creating domains where calcium concentrations felt by one single mitochondrion are much higher than in bulk cytosol [11] Also, it is known that a higher calcium amount is necessary to induce the MPTP in vitro when succinate and rotenone are present in the reaction buffer [12]. The presence of the mentioned compounds creates conditions to generate a high degree of reduction of intramitochondrial pyridine nucleotides, which antagonises MPTP induction for lower calcium matricial concentrations [12]. It is feasible that in vivo conditions, with lower ratios between reduced and oxidised intra-mitochondrial pyridine nucleotides due to mitochondrial complex I activity, a MPTP induction for much lower calcium amounts, can be observed, as it happens in isolated mitochondria (Oliveira, unpublished results).

Differences in the amounts of coenzyme Q9, Q10 and vitamin $\mathrm{E}$ in the two experimental groups were also detected. Previous works have already described increases in the amount of vitamin $\mathrm{E}$ and coenzyme $\mathrm{Q}$ in diabetic GK rats $[13,14]$, which rendered mitochondria 
from those animals more resistant to lipid peroxidation. Being the MPT, a phenomenon linked to the generation of oxidative stress inside mitochondria, our results may, in fact, seem contradictory. We believe that this is not the case. The MPT is thought to be initiated by different mechanisms than lipid peroxidation [11]. Also, an elegant study by Lass et al. [15] showed that reactive oxygen species generation is directly proportional to the rate between coenzyme Q9 and coenzyme Q10 concentrations. Facing our results, we propose that the increase in the rate between coenzyme Q9 and coenzyme Q10 seen in kidneys of GK rats (resulting from possible adaptation mechanisms) may have the result proposed by Lass et al. [15], that is, an increased generation of oxygen-free radicals by the mitochondrial respiratory chain (that would lead to the MPTP in the presence of calcium). MPTP induction would not be hindered by the increased amount of vitamin $\mathrm{E}$, whose main role would be to decrease lipid peroxidation, but not oxidative phenomena that lead to MPTP opening. As proposed in previous published works $[13,14]$, the increased oxidative stress observed in diabetic rats is probably contributing to an adaptive increase in mitochondrial amounts of coenzyme $\mathrm{Q}$ and vitamin $\mathrm{E}$. A recent investigation done in the heart mitochondria from GK rats showed a lower amount of coenzyme Q9 [16]. This was suggested to be responsible for an increased susceptibility of diabetic heart mitochondria to oxidative damage. The authors used lipid peroxidation as an index to determine the susceptibility of mitochondria to oxidative stress. As we have reported above, it is almost consensual that lipid peroxidation and the MPT are initiated and controlled through very different processes and so the role of mitochondrial antioxidants (as vitamin E and coenzyme Q) is expected to be different. Other factors that could explain differential results could be the different organs studied (different signalling processes and hierarchy of antioxidant defences) or the different age of the studied animals (26 vs 52 weeks of age).

The results presented in this work indicate that kidney mitochondria from diabetic GK rats displayed increased calcium susceptibility. The observations are of interest because they contrast with results obtained in other organs. Heart mitochondria from GK rats were shown to have increased calcium-loading capacity [17]. Also, a previous work by Kristal et al. on the susceptibility of liver mitochondria isolated from streptozotocininduced diabetic rats described a lower susceptibility to the induction of the MPTP, as compared to control mitochondria [18]. One may conclude that mitochondria from different organs may respond differently to the same pathology and that mitochondrial response to the pathological stimulus may be correlated to the extension of organ damage.

The role of the MPT in cellular dysfunction is well documented [4]. Although our results do not allow us to fully conclude this, kidney dysfunction sometimes associated with a diabetic condition can be associated with an increased susceptibility of kidney mitochondria to the permeability transition. Whatever the reason underlying the differential susceptibility to the MPT, sustained enhancement of MPTP openings in kidney mitochondria isolated from diabetic rats may be causative of a decrease in mitochondrial ATP production, large amplitude swelling as well as cell death and reduced resistance to ischemia and reperfusion [4].

Future work should focus on other animal models for diabetes, as the streptozotocin-injected rat, in order to determine if the observed effects are exclusive or not to our animal line.

In this line of reasoning, we are prone to speculate that the MPTP may be involved in the mechanisms of renal failure, as the kidney is an extremely affected organ during diabetes [2]. Although it is a highly speculative link, it is known that altered MPTP function is related to problems in mitochondrial energy production, free radical metabolism, calcium homeostasis and cell death [4]. It is fair to speculate that cell death may be enhanced in the diabetic kidney, thus contributing to the so often observed end stage of renal failure. Further work is due in this area.

\section{Acknowledgements}

We are grateful to Prof. João Patrício and co-workers (Carlos and Maria de Lourdes Silva at the Animal Research Center Laboratory, University Hospitals, Coimbra) for all work concerning animal care. We also thank Teresa Proença (Laboratory of Neurochemistry, University Hospitals, Coimbra) for the measurements of Coenzyme Q10, Q9 and Vitamin E. Paulo J. Oliveira is recipient of a fellowship from the Portuguese Fundação Para a Ciência e Tecnologia (grant PRAXIS XXI/BD/21494/99).

\section{References}

1. Flatt P, Abdel-Wahab Y, Boyd AC. Pancreatic B-cell dysfunction and glucose toxicity in non-insulin-dependent diabetes. Proc Nutr Soc 1997; 56: 243-262.

2. Pinto JR, Viberti G. The patient with diabetes mellitus. In Oxford Textbook of Clinical Nephrology, Cameron S (ed.). Oxford Medical Publication: Oxford, 1990; 93-117.

3. Broekemeier KM, Dempsey ME, Pfeiffer DR. Cyclosporin A Is a Potent Inhibitor of the inner membrane mitochondrial transition in liver mitochondria. $J$ Biol Chem 1989; 264: 7826-7830.

4. Crompton M. The mitochondrial permeability transition pore and its role in cell death. Biochem $J$ 1999; 341: 233-249.

5. Bernardi P, Colonna R, Costantini $\mathrm{P}$, et al. The mitochondrial permeability transition. Biofactors 1998; 8: 273-281.

6. Goto Y, Suzuki K, Sasaki M, Ono T, Abe S. GK rat as a model of nonobese, noninsulin-dependent diabetes. Selective breeding over 35 generations. In Frontiers in Diabetes Research: Lessons from Animal Diabetes II, Shafrir AR (ed.). John Libbey: London, 1998; 301-303.

7. Kamo N, Muratsugu M, Hongoh R, Kobatake Y. Membrane potential of mitochondria measured with an electrode sensitive to tetraphenyl phosphonium and relationship between proton electrochemical potential and phosphorylation potential in steady state. J Membr Biol 1979; 49: 105-121.

8. Rajdev S, Reynolds IJ. Calcium Green-5N, a novel fluorescent probe for monitoring high intracellular free $\mathrm{Ca}^{2+}$ concentrations associated with glutamate excitotoxicity in cultured rat brain neurons. Neurosci Lett 1993; 162: 149-152.

9. Takada M, Ikenoya S, Yuzuriha T, Katayama K. Simultaneous determination of reduced and oxidized ubiquinones. Methods Enzymol 1984; 105: 147-155. 
10. Takayanagi R, Takeshige T, Minakami P. NADH- and NADPHdependent lipid peroxidation in bovine submitochondrial particles. Biochem $J$ 1980; 192: 853-860.

11. Kowaltowski AJ, Castilho RF, Vercesi AE. Mitochondrial permeability transition and oxidative stress. FEBS Lett 2001; 495: $12-15$.

12. Rizzuto R, Pinton P, Brini M, Chiesa A, Filippin L, Pozzan T. Mitochondria as biosensors of calcium microdomains. Cell Calcium 1999; 26: 193-199.

13. Santos MS, Duarte AI, Matos MJ, Proenca T, Seica R, Oliveira CR. Synaptosomes isolated from Goto-Kakizaki diabetic rat brain exhibit increased resistance to oxidative stress: role of vitamin E. Life Sci 2000; 67(25): 3061-3073.

14. Santos MS, Santos DL, Palmeira CM, Seica R, Moreno AJ, Oliveira CR. Brain and liver mitochondria isolated from diabetic
Goto-Kakizaki rats show different susceptibility to induced oxidative stress. Diabetes Metab Res Rev 2001; 17(3): 223-230. 15. Lass A, Kwong L, Sohal RS. Mitochondrial coenzyme Q content and aging. Biofactors 1999; 9: 199-205.

16. Santos DL, Palmeira CM, Seica R, et al. Diabetes and mitochondrial oxidative stress: a study using heart mitochondria from the diabetic Goto-Kakizaki rat. Mol Cell Biochem 2003; 246(1-2): 163-170.

17. Oliveira PJ, Rolo AP, Seiça R, Palmeira CP, Santos MS, Moreno AJ. Decreased susceptibility of heart mitochondria from diabetic GK rats to mitochondrial permeability transition induced by calcium/phosphate. Biosci Rep 2001; 21: 55-62.

18. Kristal BS, Matsuda M, Yu BP. Abnormalities in the mitochondrial permeability transition in diabetic rats. Biochem Biophys Res Commun 1996; 222: 519-523. 